\title{
ON A THEOREM OF LINDELÖF CONCERNING PRIME ENDS
}

\author{
T. M. FLETT
}

(Received April 28, 1956)

A short proof of the following well known theorem of Lindelöf has been given by Tsuji [2].

THEOREM. Let $D$ be a bounded simply-connected domain, and let $w=f(z)$ map $D$ conformally on $|w|<1$. If $\left\{z_{n}\right\}$ is a sequence of points of $D$ such that the sequence $w_{n}=f\left(z_{n}\right)$ converges to a point $\alpha$ of $|w|=1$ in a Stolz angle, $|\arg (\alpha-w)|<\frac{1}{2} \pi-\delta$, then every limit-point of the sequence $\left\{z_{n}\right\}$ is a principal point ${ }^{1)}$ of the prime end of $D$ which corresponds to $\alpha$.

In this note we show how the proof of the theorem may be simplified still further by using a very elementary topological argument.

Tsuji proves the theorem by combining the following results.

LEMmA A. Let Dba bounded simply-connected domain, and let $w=f(z)$ map $D$ conformally on $|w|<1$. Let $\left\{\rho_{n}\right\}$ be a sequence of positive numbers such that $\rho_{n+1} \leqq \frac{1}{2} \rho_{n}<1$, and let $S_{n}$ be the domain $\frac{1}{2} \rho_{n}<|1-w|<\rho_{n}$, $|w|<1$. Then we can find an increasing sequence $\left\{n_{\nu}\right\}$ of positive integers, and a chain $\left\{q_{\nu}\right\}$ of cross-cuts of $D$ associated with the prime end of $D$ which corresponds to $w=1$, such that the image of $q_{v}$ in $|w|<1$ is an arc of a circle with centre $w=1$ lying in $S_{n v}$.

LеммA B. Let $D$ be a bounded simply-connected domain, $F(D)$ its frontier, let $w=f(z)$ map $D$ conformally on $|w|<1$, and let $z=\psi(w)$ be the inverse of $f$. Let $\left\{\rho_{n}\right\}$ be a sequence of positive numbers such that $\rho_{n+1} \leqq \frac{1}{2} \rho_{n}<1$, and let $T_{n}$ be the domain $\frac{1}{2} \rho_{n}<|w-1|<\rho_{n},|w|<1,|\arg (1-w)|<\frac{1}{2} \pi-\delta<$ $\frac{1}{2} \pi$. Then we can find an increasing sequence $\left\{n_{\nu}\right\}$ of positive integers such that the values of $\psi(w)$ in $\bar{T}_{a_{\nu}}$ converge to a point a of $F(D)$.

The deduction of the theorem from these two lemmas is immediate. For we may suppose that $\alpha=1$, and that $\left\{z_{n}\right\}$ converges to a point $a$ of $F(D)$. We can then find a chain of cross-cuts $\{\boldsymbol{q}$,$\} , associated with the prime end$ of $D$ which corresponds to $w=1$, converging to the point $a$, and this is the required result.

1) For the definition of this and other terms belonging to the theory of prime ends, see Carathéodory [1]. 
We have nothing new to add concerning the proof of Lemma $\mathrm{A}$. In place of Tsuji's proof of Lemma B (which uses Lemma A), we have, however, the following argument.

We may evidently suppose $\rho_{0}$ so small that each of the domains $U_{n}$ defined by the relations

$$
\frac{1}{4} \rho_{n}<|1-w|<2 \rho_{n}, \quad|\arg (1-w)|<\frac{1}{2} \pi-\frac{1}{2} \delta
$$

is completely contained in $|w|<1$. Let

$$
\psi_{n}(w)=\psi\left\{1+\rho_{0} \rho_{n}^{-1}(w-1)\right\}
$$

The functions $\psi_{n}$ are regular and uniformly bounded in $U_{0}$, and the values taken by $\psi_{n}$ in $T_{0}$ and $U_{0}$ are the values taken by $\psi_{\text {in }} T_{n}$ and $U_{n}$ respectively. By Montel's theorem, we can select a subsequence of the $\psi_{n}$ which converges uniformly in any closed set in $U_{0}$, and, in particular, in $\bar{T}_{0}$, to a regular $\phi$. If $\phi$ is not constant in $U_{0}$, the values taken by $\phi$ in $U_{0}$ form an open set. This set contains the area of some complete circle, and this is impossible, since it is contained in $F(D)$. Hence $\phi$ is constant in $U_{0}$, and this proves the lemma.

\section{REFERENCES}

[1] C CAR ATHÉODORY, Über die Begrenzung einfach zusammenhangender Gebiete, Math. Ann., 73(1913), 323-370.

[2] M. Tsuji, On the theorems of Carathéodory and Lindelöf in the theory of conformal representation, Jap. J. Math, 7(1930), 91-99.

UNIVERSITY OF LIVERPOOL. 